\title{
Le silence, mise à l'épreuve de la sincérité au théâtre (1802-1828)
}

Héritier d'une conception rousseauiste du langage, le mélodrame, art théâtral qui connaît un immense succès sous le Consulat et l'Empire en France, assigne au sonore (il contient souvent des couplets chantés, comme le rappelle son étymologie), mais aussi au silence un statut et une fonction plus nobles qu'au discours : dans cette dramaturgie à l'axiologie clivée, les personnages de traîtres, de criminels, d'hommes politiques puissants mentent, manipulent les mots avec adresse, tandis que ce sont ceux qui se taisent, par timidité, décence ou sensibilité, qui ont la faveur de pièces exhibant le silence en tant que valeur éthique. Il s'agit également d'une valeur esthétique : pour ses spectateurs postrévolutionnaires friands de situations spectaculaires qui laissent, littéralement, sans voix, le silence mélodramatique est un instrument de mesure de l'effet. Un mélodrame réussi propose à chaque acte des tableaux muets saisissants dans lesquels des personnages "parlent » uniquement avec leurs corps, dans un registre qui emprunte à la pantomime mais aussi à la gestuelle opératique. Le cinéma muet s'en souviendra, comme le prouve le nomadisme générique des plus grands succès du mélodrame : pour ne donner qu'un seul exemple, Les deux orphelines d'Adolphe d'Ennery et Eugène Cormon jette, en 1874, sur la scène du théâtre parisien de la porte Saint Martin, ses derniers feux, mais l'intérêt pour son intrigue à multiples rebondissements ne faiblit pas, puisqu'adapté par ses auteurs en 1877 en roman, il obtient un succès considérable, relancé par de nombreuses rééditions, notamment sous forme de feuilleton (dans La Nation, en 1892) ou de collections populaires. Puis, il fait l'objet d'adaptations cinématographiques, dont l'un des derniers chefs-d'œuvre du cinéma muet nord-américain (sous le titre Orphans of the Storm, D. W. Griffith, 1921). Dans ces récits, le silence bâtit un espace essentiel à la compréhension : toujours temporaire, mais marquant, il stimule le débat, appelle l'aveu et l'exposition de la vérité. Les moments de mutisme abondent : des personnages cachés, néanmoins ostensiblement portés à la vue du spectateur, ne font aucun bruit

Florence Fix - professeur en littérature comparée à l'Université de Rouen-Normandie. Adresse pour correspondance : Université de Rouen, UFR de Lettres et sciences humaines, bureau A 305, rue Lavoisier, 76821 Mont Saint Aignan ; e-mail : florence.fix@gmail.com 
afin d'écouter les projets de criminels. D'autres se mêlent aux protagonistes malfaisants, mais feignent temporairement d'être muets pour ne pas éveiller leur vigilance ; d'autres enfin, muets par accident ou par agression, trouvent toutefois moyen, grâce à l'expressivité de leur gestuelle, de faire connaître leurs déboires et d'en obtenir réparation. Le silence " parle » et communique des informations et des valeurs essentielles.

\section{Dire vrai}

Dans cette construction très polarisée - aux bavards le mensonge, aux muets la vérité - des espaces de porosité et de déplacement existent aussi : il arrive que les traîtres et les criminels pratiquent le non-dit, laissent leurs phrases en suspens et fassent grand usage des points de suspension, mais la répartition des valeurs ne bouge pas. Un criminel ne garde le silence que par opportunisme ou pour fomenter quelque mauvais coup supplémentaire ; les personnages vertueux, au contraire, ont le silence bavard et utile à la collectivité : dénués de la capacité de parole, ils écrivent des lettres qui, lues tour à tour par plusieurs protagonistes, agissent comme autant de relances de l'information et de l'action, ou bien ils font de grands gestes explicites de mise en garde d'autrui, ou même recouvrent opportunément leur voix, sous le coup d'une émotion forte ou d'une urgence vitale.

Dans le mélodrame, tout est outrancier, des situations stéréotypées aux personnages excessifs, des éruptions volcaniques aux torrents déchaînés qui emportent les traîtres : ce genre du panache et du rythme, dont on trouve l'héritage dans le drame romantique, donne certes la part belle à l'emphase, mais n’en déplaise à Émile Zola, n'est pas seulement gonflé de tirades grandiloquentes et de « personnages en pourpoint qui font les grands bras et qui s'agitent comme des hannetons grisés de soleil » (1881: 8). Il déploie également des moments de silence que la didascalie décrit très précisément, empruntant les mots du discours, comme dans cet exemple issu du Pèlerin blanc: "Le comte paraît attendri; Roland le prend fortement par le bras, et lui ordonne impérieusement de se retirer. Le comte sort après avoir montré tout l'intérêt qu'il prend aux enfants, et la résolution où il est de les sauver. " (Pixerécourt, 1971a : 132).

De cette façon, alors que dans le théâtre classique « le langage s'affirme nettement comme un indice de pouvoir, qui fait du silence la marque d'une dévalorisation du personnage » (Rykner, $1996: 36)$, le mélodrame invite à une lecture inverse : certes, les puissants ont la parole ; dotés d'un verbe performatif, ils gouvernent, décident. Mais le développement de l'intrigue leur donne tort et ce sont les silencieux sans pouvoir que l'issue de la pièce révèle comme porteurs de vérité. Conformément aux écrits alors en vogue de lecture de l'expression des visages, notamment ceux de Lavater, traduits de l'allemand et publiés en français entre 1806 et 1809 sous le titre Physiognomonie ou l'art de connaitre les hommes, un front large, un regard clair sont le gage d'une personnalité qui ne ment pas. Nul n'est besoin de parler, le visage s'ex- 
prime. Ainsi de ce dialogue entre un maitre de maison et sa servante dans le plus grand succès du dramaturge Pixerécourt, Coelina ou l'enfant du mystère : le premier veut chasser un muet indigent, la seconde prend sa défense :

Dufour. [...] Je voudrais bien savoir quel intérêt vous prenez tous à un mendiant que vous ne connaissez pas plus que moi, et qui a abusé de ma sensibilité pour s'introduire ici et s'y établir?

Tiennette. Quel intérêt, Monsieur ? celui que l'on prend à tous les malheureux. Je ne sais qui il est, cet homme; j'ignore jusqu'à son nom, mais il a une physionomie si douce, il jette sur moi des regards si expressifs qu'on ne peut s'y méprendre. Oui, Monsieur, je m’y connais, je vous réponds que c'est un honnête homme et qu'il a éprouvé de grands malheurs.

Dufour. Qui te l'a dit?

Tiennette. À coup sûr, ce n'est pas lui, puisqu'il est muet ; mais sa profonde tristesse me l'assure. (1971a : 19).

Le corps muet « parle » clairement et se révèle à qui sait le lire. De ce fait, l'axiologie positive qui touche le mutisme s'étend à ceux des protagonistes qui, loin de le dénigrer ou de l'ignorer, en déchiffrent les signes. Dans la construction morale très discriminante - propre au mélodrame, les " mauvais » personnages sont brutaux, bruyants et dédaigneux des faibles, quand les «bons » sont en empathie immédiate avec les silencieux. Colina ne se trompe pas à l'épreuve du silence, et le spectateur ne se trompe pas non plus sur les qualités de la jeune fille :

Célina. Souvent je le vois me regarder fixement et cherchant à lire dans mes yeux ce qui m’occupe ou m'intéresse. Quand il croit l'avoir deviné, il me quitte et revient bientôt m’apporter ce qu'il suppose être l'objet de mes désirs. Lorsqu'il a réussi, la joie la plus vive brille sur son visage ; il semble tout fier d'avoir pénétré ma pensée, et me demande d'un air suppliant de lui permettre de baiser ma main qu'il baigne de ses larmes. O mon oncle ! on ne peut être un méchant homme avec un si bon cœur. (Pixerécourt, 1971a : 22).

La plupart des mélodrames scéniques sont des adaptations de romans-fleuves à succès (à l'exemple de la pièce Coelina, d'après le roman noir de François-Guillaume Ducray-Duminil, publié en 5 volumes, en 1798). Les « faiseurs » qui s'y emploient doivent donc ôter les longues descriptions tout en conservant la morale du visible essentielle autant à leur structure narrative quà leur éthique de lecture : il faut que le lecteur se sente flatté par un spectacle qui exhibe des personnages puissants, adroits en actes comme en discours, mais place à l'arrière-plan des maladroits dont il a l'impression d'être seul à déceler l'importance. Dans cette optique consolatoire, le mélodrame n'est pas avare en signes et sature le mutisme d'indications valorisantes. D'une part, les muets déploient une gestuelle qui doit beaucoup à l'iconographie religieuse édifiante (mains jointes, regards émus portés vers le ciel, larmes silencieuses, génu- 
flexions ou agenouillements...) ; d'autre part, leurs adjuvants décrivent avec emphase en leur absence leur comportement exemplaire, comme on vient de le voir à l'exemple de Coelina décrivant le muet.

Leçon donnée aux puissants, le mélodrame pratique une redistribution des valeurs très imprégnée de morale chrétienne : aux bavards et aux menteurs, une gloire éphémère, que l'issue des pièces se charge de sanctionner par un décès spectaculaire ; aux muets et aux humbles, un doux triomphe final, dans l'assentiment général sous forme d'harmonie familiale et sociale retrouvée. Si les «bons » personnages se taisent (temporairement) aucune mauvaise action n'est passée sous silence : in fine, l'outrance argumentative du silence a raison de toutes les malversations familiales et de tous les complots politiques. À l'instar de la justice distributive biblique qui promet le paradis aux pauvres d'esprit, le mélodrame promet aux pauvres de discours la reconnaissance de leurs qualités et de leurs droits, non sans avoir traversé un mutisme construit comme une épreuve de la volonté et de la constance : le banni Hernani qui tait sa véritable identité jusqu’à l'avant-dernier acte de la pièce de Victor Hugo (1830), le laconique et discret comte de Monte-Cristo d'Alexandre Dumas (1844), entre autres, en sont les continuateurs.

\section{Contre-pouvoir}

Le silence à double entente s'élabore comme une entreprise de sous-conversation : le public assiste, dans le plaisir de son propre silence imposé (et pas toujours respecté, car le spectateur du célèbre Boulevard du Crime parisien est fameux pour ses cris, ses adresses à l'encontre des acteurs et ses manifestations bruyantes), à l'agile gestuelle de personnages muets qui contrent habilement mais modestement le déploiement paroxystique de menaces et d'injustices des traîtres, finalement inopérantes. Le silence est un espace qui permet de juxtaposer en même temps plusieurs discours et plusieurs lectures, ainsi qu'en témoigne cette scène muette de Le Belvédère ou la Vallée de l'Etna:

Au moment où Floretta a été forcée de se tourner à gauche, Léonard est venu se placer vivement entre elle et sa maitresse, un peu en arrière, et s'est adroitement saisi de la lettre, de manière qu'elle doit croire que c'est Emilia qui l'a prise ; puis il s'est retiré bien vite pour venir à la droite de la jeune Duchesse. Dans le même moment, celle-ci a été forcée de regarder à droite, parce que Léonard a fait avancer un de ses compagnons qui présente son chapeau pour demander une aumône. Ce jeu de théâtre, très vif, ne peut être aperçu que des pirates qui ont intérêt à ne pas le faire remarquer. (Pixerécourt, 1971b : 527).

Paradoxalement, le mutisme autorise une certaine liberté de mouvement et d'action pour des personnages qui échappent ainsi, un temps, à leurs persécuteurs ; parce qu'ils apparaissent fragiles, de faible importance, les muets vont partout, écoutent 
tout, sans être inquiétés. Et si les arrogants puissants les ignorent (à tort), le spectateur est constamment en connivence avec eux : à l'aide de nombreux soupirs, phrases interrompues, gestes arrêtés, larmes et yeux tournés vers le ciel, le protagoniste muet montre ostensiblement au spectateur qui il est vraiment. Les scènes muettes sont auto-suffisantes et sans détours, comme dans cette didascalie de L'Homme à trois visages: "Ici, on voit Orsano traverser le théâtre de gauche à droite et paraissant suivre des yeux les mouvements de quelqu'un : il indique suffisamment que c'est Vivaldi qu'il épie. " (Pixerécourt, 1971a : 210). Au besoin du reste, les répliques d'un personnage viennent conforter les gestes d'un muet, manifestant la cohérence des opinions et l'harmonie des sentiments que le silence abrite. Ainsi de cet "échange » dans Le Chien de Montargis entre une jeune fille et un serviteur muet qui tient de l'aimable pantomime de pastorale :

URSULE. À merveille, mon petit Éloi. C’est très joli, n'est-ce pas?

(Éloi fait entendre à Ursule qu'il ne peut rien sortir que d'ingénieux de sa bouche ou de sa pensée.)

Ursule. Tu es un flatteur. Si je te croyais, tu finirais par me persuader que je suis une petite merveille.

(Éloi fait entendre à Ursule que c'est précisément là ce qu'il a voulu dire.)

(Pixerécourt, 1971b : 126).

Le puissant ne se méfie pas assez du muet : l'outrance verbale du traitre se révèle verbiage indigne, le mutisme, lui, action efficace en faveur de la vérité. Aussi l'aveu verbal final des traîtres et des criminels, indispensable à la conclusion heureuse d'un mélodrame, s'il éveille la consternation pour certains des protagonistes scéniques, ne peut être que confirmation pour les spectateurs qui n'en avaient pas douté. Il y a là une délectation du silence, qui s'offre comme un espace de contre-pouvoir, voire de pouvoir véritable : alors que les traîtres et autres faussement puissants s'agitent sur scène en des tirades inutiles, le muet affirme en contrepoint sa fidélité constante. Le bavard ment, le muet tient parole. C’est pourquoi le silence assure à la pièce une forme de continuité, quand la grandiloquence des traîtres est toujours disruptive : il confirme ce qui vient d'être dit (que les interlocuteurs soient muets d'admiration, ou trop émus pour répondre) ou bien, quand il exprime une opposition (les interlocuteurs, révoltés, indignés cherchent leurs mots pour contrecarrer), il maintient toujours, en termes de contenu, une cohérence avec les valeurs défendues par la pièce. Le mutisme constitue une pause qui dramatise le dialogue mais n'est nullement exogène à la fable. Le contre-discours qu'il promeut invite à une double lecture continue et efficace : à l'avant-scène, de beaux parleurs croient détenir un pouvoir dont ils abusent par le biais de menaces, interdictions, humiliations ; à l'arrière-scène, une autre scène se joue : les bannis, les proscrits, les humbles et les faibles par une ges- 
tuelle très visible affirment la permanence de leurs valeurs de fidélité, d'honnêteté et la prééminence de l'émotion. Le silence est ainsi l'espace d'affirmation d'une expérience sensible du monde, à l'encontre de la rhétorique du pouvoir : les pauses muettes sont autant de moments qui préparent efficacement le dénouement, qui voit toujours le triomphe des faibles contre les traitres.

Le mélodrame est en effet un théâtre des émotions fortes et des rebondissements nombreux, hérités du gothic novel britannique : pour assurer le suspense, la divulgation de l'information est souvent différée et fragmentée par étapes. Les personnages «bons » attendent le moment pertinent pour dévoiler les injustices dont ils ont souffert et pratiquent un mutisme temporaire. Les " mauvais ", eux, utilisent le silence comme menace : dans Coelina ou l'enfant du mystère, l'arriviste Truguelin sort de scène en silence à la fin de l'Acte I, non sans avoir annoncé que sa reprise de parole serait dangereuse : " un seul mot peut rompre le mariage que vous projetez, et ce mot je le dirai. Adieu. (Il sort avec Germain.) "(Pixerécourt, 1971a : 38). Dramaturgie du complot permanent, le mélodrame installe une scène sous surveillance : les personnages cachent leurs identités, comme le promettent des titres tels que L'homme à trois visages ou La femme à deux maris. Le silence n'est pas un moment de pause ou de repos, mais un temps de saturation de l'anxiété, qui convoque souvent des scènes à tiroirs : un personnage parle à un second, lui-même espionné par un troisième, et seul le spectateur connaît toutes les identités et toutes les intentions des personnages. La gestuelle silencieuse lui est destinée et a la fonction d'un commentaire auctorial romanesque visant à l'informer : "Pendant toute cette scène, Vivaldi tourne souvent la tête pour voir s'il est toujours observé : alors Orsano se retire, mais pas assez vite pour n'être pas aperçu quelquefois. Il donne de fréquentes marques d'approbation au discours de Vivaldi.» (Pixerécourt, 1971a : 210). En outre, la rupture du silence, dans ces dramaturgies du mystère et du non-dit, provoque de saisissants coups de théâtre que prépare une surenchère d'effets d'annonce : à de multiples reprises, le traitre de La Femme à deux maris menace l'héroïne de dévoiler à tous qu'elle est bigame ; à l'inverse, les « bons " personnages attendent le moment favorable pour dévoiler identité, filiation ou complot. Le silence et ses multiples usages (garder un secret, feindre d'être muet, taire ses intentions ou son identité, etc.) constituent donc, dans cette dramaturgie, un point de tension permanent et un instrument de pouvoir : celui qui habilement se tait sait in fine se faire entendre.

\section{Le muet et le spectacle}

Au cœur de cette utilisation stratégique du silence, un personnage récurrent retient l'attention : le muet ou la muette (dont on connaît le succès jusqu'en 1828 avec l'opéra d'Auber La Muette de Portici qui, dit-on, engendra la révolution belge de 1830), figure emblématique à lire selon le principe de la blessure compensatoire. Son infirmité, en effet, le ou la rend encore plus éloquent(e) : son corps très mobile sur la scène 
de théâtre, son visage animé d'expressions fortes assurent autant de sa sincérité que de sa pertinence. En d'autres termes, il est aussi bien fidèle aux personnages injustement maltraités qu'il défend et contribue à sauver, qu'aux valeurs du mélodrame dont il est l'indéfectible " porte-parole ». Le muet (qui peut même être un animal, comme dans Le Chien de Montargis de Pixerécourt, où le fidèle compagnon d'un homme assassiné désigne son meurtrier) ne se trompe jamais et dénonce inlassablement les crimes et les complots. Il peut même, dans ce but, feindre le handicap : il y a ainsi un faux muet dans Les Ruines de Babylone (il s'agit du vizir injustement banni par le calife), dans Le Pèlerin blanc (un père qui protège discrètement ses fils), et plus généralement de nombreux moments où un personnage choisit de se taire.

Dans sa variante touchante, il est interprété par une femme, alors louée par le public pour son expressivité (c'est le cas du jeune Éloi, commissionnaire muet, dans Le chien de Montargis, joué par une comédienne), dans sa variante plus politique (un exilé feint d'être muet pour recouvrer ses droits et confondre les traîtres), il donne l'occasion à des comédiens célèbres de déployer une grande variété de jeu. Il y a là une forme de contradiction : le silence récuse l'histrionisme et l'artifice, mais il amène des comédiens à surjouer la surprise, le désaccord, la compassion - postures qui leur valent les éloges de la critique théâtrale dans la presse de l'époque. Le jeu silencieux, qui prétend être un mode mineur, est en fait souvent spectaculaire. Aussi considérera-t-on comme fausse modestie la didascalie suivante :

Éloi répond par la pantomime la plus expressive, et lui fait entendre que ce n'est pas de la reconnaissance, mais de l'amour, et l'amour le plus vif qu'il ressent pour elle.

URSUle. Ce n'est pas ce que je vous demande, Monsieur; vous êtes un babillard.

(Éloi voudrait bien que sa bouche pût exprimer tout ce que lui inspire Ursule; mais la pantomime est impuissante et ne peut être qu'un faible interprète des sentiments dont son cour est rempli.)

(Pixerécourt, 1971b : 127).

Alors que les débats sur les paradoxes du comédien de Diderot, poursuivis par le traité de Riccoboni en 1738, se poursuivent au cours du XIX ${ }^{e}$ siècle, l'engouement du public parisien pour le mélodrame rappelle que le comédien ne doit pas faire sensation mais produire du sensible. Il s'agit d'en appeler à l'émotion, de pleurer, de soupirer, de savoir se taire : ces spectateurs, éprouvés par la Révolution et la Terreur, font montre d'une certaine réticence envers l'emphase qui a été l'apanage d'un discours politique belliqueux. Ils lui préfèrent le langage d'un corps muet mais expressif, renouant avec une iconographie religieuse ancienne qui donne la part belle aux gestes de compassion qui « disent » directement les élans du cœur et ne les travestissent pas par un langage de culture savante. Le jeu du tragédien Mounet-Sully qui triompha dans les grands rôles raciniens, à la fin du XIX $x^{\mathrm{e}}$ siècle, du comédien Coquelin l'Aîné qui créa en 1897 le rôle du Cyrano de Rostand qui lui dédia la pièce, ou de la grande Sarah Bernhardt, la " voix d'or » selon Victor Hugo, dans Ruy Blas en 1872, en sont 
les héritiers. La diction théâtrale exige alors de la sensibilité plus que de la virtuosité : ponctuée de silences inspirés, appuyée sur une gestuelle vive, elle ne repose pas exclusivement sur le verbe et parfois le désacralise. Ainsi, aussi paradoxal que cela puisse paraître à première vue, autour de 1800 et jusqu'au drame romantique à tout le moins, pas d'art déclamatoire sans silence. Dans ses Réflexions sur l'art théâtral, en 1801, Jean Mauduit-Larive regrette la construction à Paris d'édifices de spectacle de grande ampleur qui contraignent selon lui les comédiens à crier pour être remarqués et la disparition progressive de petites salles qui mettaient en valeur un jeu plus délicat. À propos de l'ancienne salle du Faubourg Saint-Germain, il écrit :

Cette salle avait les justes proportions favorables à tous les accents de la voix ; et ces accents ne perdaient rien de leur charme dans les intonations, même les plus moelleuses et les plus simples; les spectateurs y jouissaient sans effort et sans inquiétude : le théâtre, plus facile à éclairer, permettait de tout voir, et la scène muette n'était pas perdue comme elle l'est aujourd'hui. (Mauduit-Larive, $1801: 6$ ).

On constate donc que la question de l'usage du silence au théâtre (et de ses déclinaisons de la voix comme le chuchotement, l'intonation faible, le soupir...) dépasse largement le cadre du seul mélodrame, dont le caractère reste emblématique en raison de son grand succès et de son usage massif d'une part, du mutisme comme ressort dramatique et, d'autre part, du muet comme héros ordinaire. Il induit un débat d'idées sur le jeu d'acteur, en proposant une critique de l'art déclamatoire ainsi que du verbe théâtral stylisé. Lieu d'une expression extrêmement visible, le silence théâtral se donne à lire comme un pacte de lecture ambigu qui invite le spectateur à consentir à l'illusion et à l'empathie tout en l'invitant à une distance critique envers la grandiloquence. Un adroit discours, bien agencé, séduisant et convaincant doit être lu dans l'axiologie du mélodrame comme marque d'artifice et d'insincérité. À l'inverse, un discours malhabile, hésitant sous le coup de lémotion ou impossible en raison du handicap d'un protagoniste qui reste muet mais pas inexpressif, se déclare aux yeux des spectateurs comme essentiellement véridique et sincère. Le bavard est culturellement et politiquement dominant, il abuse souvent de son pouvoir : ce sont les figures honnies alors du roi, du courtisan, du ministre corrompu, du séducteur beau parleur, du traitre manipulateur. Le muet est impuissant, mais tendre et modeste ; son silence ne l'empêche pas de se faire entendre ; au contraire, le spectateur est invité à lui prêter la plus grande attention. Le muet émarge au registre des benêts au grand cœur, des serviteurs bafoués, des enfants dont l'héritage a été usurpé, mais aussi des bannis réduits au silence, il est le porte-parole sur scène des vies minuscules.

Le mélodrame n'est pas un théâtre de la distance et encore moins de la distanciation : c'est un théâtre de la proximité revendiquée qui sollicite constamment l'empathie du 
spectateur. Espace du sensible, récusant ostensiblement toute intellectualisation, ce qui a pu lui faire reprocher de se constituer en forme conservatrice voire réactionnaire, le mélodrame s'appuie sur le silence comme expression de l'émotion sincère.

Pour réussir à cet égard, il faut qu'un geste, un regard puisse être saisi sans être forcé, et que le jeu de la physionomie, avant-coureur des expressions de l'âme, soit vu également de tous les spectateurs.

La scène muette soutient l'intérêt de la représentation, et contribue le plus à son ensemble. (Mauduit-Larive, $1801: 7$ ).

Le silence n'est pas ici un élément d'incertitude ou d'embarras, il maintient un lien cohérent entre les scènes, il rappelle régulièrement, au sein d'une intrigue échevelée, riche en rebondissements et en quiproquos, que les valeurs d'honnêteté, de générosité triompheront à l'issue de la pièce. Il donne une leçon de jeu d'acteur et une leçon d'analyse du discours : il engage le spectateur à se défier des effets d'emphase et des parlures artificielles pour l'encourager à distinguer les nuances des intonations et des gestes.

\section{BIBLIOGRAPHIE}

Mauduit-Larive J. 1801. Réflexions sur l'art théâtral. Paris. Rondonneau.

Pixerécourt R. C. G. de. 1971a. Théâtre choisi. Tome 1. Genève. Slatkine.

Pixerécourt R. C. G. de. 1971b. Théâtre choisi. Tome 3. Genève. Slatkine.

Rykner A. 1996. L'envers du théâtre. Dramaturgie du silence de l'âge classique à Maeterlinck. Paris. José Corti.

Zola É. 1881. Le théâtre naturaliste. Paris. Charpentier.

\section{Testing and contesting authority: silence in French theatre (1802-1828)}

ABSTRACT: In $19^{\text {th }}$ century French very successful melodrama, discourse is seen as a powerful way to get attention. Criminals and traitors are masters of speech and seductive persuasion, but they never achieve their goals by the end of the play. On the other hand, the mute, humble and shy characters demonstrate that silence can convey values such as honesty, loyalty and show the audience that sensibility should be preferred to histrionic actor play.

Keywords: melodrama, mute characters, pantomime, mutism, Pixerécourt. 